\title{
Determination of Rocuronium in Human Plasma by High Performance Liquid Chromatography-Tandem Mass Spectrometry and its Pharmacokinetics in Patients
}

\author{
Yuan Guiyan, Zhang Rui, Wang Benjie, Wei Chunmin, Liu Xiaoyan, Zhao Wenjing and Guo Ruichen ${ }^{\text {* }}$ \\ Institute of Clinical Pharmacology, Qilu Hospital of Shandong University, Jinan Shandong, China
}

\begin{abstract}
A sensitive and selective high performance liquid chromatography-tandem mass spectrometric (HPLC-MS/MS) method was developed and validated for the determination of rocuronium in human plasma. The plasma samples were separated on an Agilent SB-C ${ }_{18}$ column $(150 \times 2.1 \mathrm{~mm}, 3.5 \mu \mathrm{m})$ with a mobile phase consisted of $20 \mathrm{mM}$ ammonium acetate/methanol/ acetonitrile $(20 / 40 / 40, \mathrm{v} / \mathrm{v} / \mathrm{v})$ at a flow rate of $0.7 \mathrm{~mL} \cdot \mathrm{min}^{-1}$. Electrospray ionization (ESI) source was applied and operated in the positive ion mode. Multiple Reaction Monitoring (MRM) modes with the transitions of $\mathrm{m} / \mathrm{z} 529.3 \rightarrow 487.3$ (rocuronium) and $321.0 \rightarrow 275.0$ (IS) was used. A good linearity was obtained in the concentration range of $5 \sim 3000 \mu \mathrm{g} \cdot \mathrm{L}^{-1}(\mathrm{r}=0.9976, \mathrm{n}=5)$. The inter- and intra-day precision (RSD) were less than $8.1 \%$. The extraction recoveries were $92.0 \sim 92.6 \%$. Rocuronium in plasma was stable when frozen at $-20^{\circ} \mathrm{C}$ for 24 hours and seven days, and also stable after two freeze-thawing cycles. The method is simple, quick, sensitive, reproducible and can be used for the pharmacokinetic and bioequivalence study of rocuronium.
\end{abstract}

Keywords: Rocuronium; High performance liquid chromatographytandem mass spectrometry; Pharmacokinetics

\section{Introduction}

Rocuronium, a new type of muscle relaxant with the features of single quaternary ammonium steroids, medium aging and non depolarizing, was used for anesthesia and surgery of endotracheal intubation of muscle relaxation, which has quick effect, short duration and no cumulative effect, and do not produce tachycardia and blood pressure change, no histamine release, and other features. It is one of the most widely used muscle relaxants at present $[1,2]$.

Less studies were reported on the determination of rocuronium. $\mathrm{Yu}$ et al. [3] reported a gas chromatography mass spectrometric (GCMS) method for determination rocuronium in human plasma, which has higher limit of quantitation and longer time for sample analysis. Shi et al. [4] developed a high performance liquid chromatography with fluorescence detection (HPLC-FD) method to determine the concentration of rocuronium in patient's plasma, which sample treatment was time-consuming because of rocuronium derivatization. Farenc et al. [5] established a high performance liquid chromatographymass spectrometric (LC-MS) method to determine the concentration of rocuronium in human plasma. In this method, the limit of quantitation is higher $\left(25 \mathrm{ng} \cdot \mathrm{mL}^{-1}\right)$, and trifluoroacetic acid was added to the mobile phase which can damage the ion source because it is less volatile. The aims of this paper were to establish a LC-MS/MS method to determine rocuronium in human plasma and to study the pharmacokinetics of rocuronium in patients used the established method.

\section{Experimental}

\section{Chemical, regents and instruments}

Rocuronium standard (purity $>99 \%$, Batch number 63254132) was provided by Organon Pharmaceutical Co Ltd (Oss, Netherlands). Lorazepam standard (Batch number 171253-200401) was provided by national institutes for food and control. HPLC grade methanol and acetonitrile (Batch number LOTG06E10 and LOTE30818) were purchased from J.T. Baker Company (USA). Analytical pure ammonium acetate (Batch number 060223) was provided by Chemical Industry Institute of Shandong Province (Jinan, China).
The HPLC components consisted of an Agilent 1200 liquid chromatography system with a G1312B binary pump, G1379B degasser, G1316B thermostated column compartment and G1367C auto-sampler (Agilent Technologies, USA). An Agilent 6410 triple quadrupole mass spectrometer equipped with a Turbo Ion Spray (ESI) source was used for mass analysis and detection (Agilent Technologies, USA).

\section{Chromatographic conditions}

Chromatographic separation was performed using a Agilent $\mathrm{SB}_{18}$ column $(3.5 \mu \mathrm{m}$ particle size, $150 \times 2.1 \mathrm{~mm}$ internal diameter; Agilent Technologies). The isocratic mobile phase consisted of methanol/ acetonitrile/20 $\mathrm{mM}$ ammonium acetate buffer solution (20/40/40, $\mathrm{v} / \mathrm{v} / \mathrm{v})$. The flow rate of the mobile phase and the column oven temperature were set at $0.7 \mathrm{~mL} / \mathrm{min}$ and $20^{\circ} \mathrm{C}$, respectively.

\section{Mass spectrometric conditions}

The ESI source was set to positive ion mode and multiple reaction monitoring (MRM) mode was used to detect rocuronium and lorazepam (internal standard, IS) at the transition of $529.3 \rightarrow 487.3$ and $321.0 \rightarrow 275.0$, with spray gas pressure of $40 \mathrm{psi}$, protective air of nitrogen gas at a flow rate of $9 \mathrm{~L} / \mathrm{min}$, capillary voltage of $4000 \mathrm{~V}$, fragment electric voltage of $100 \mathrm{~V}$, and collision energy of $45 \mathrm{eV}$ for rocuroniumand $20 \mathrm{eV}$ for I.S.

*Corresponding author: Guo Ruichen, Institute of Clinical Pharmacology, Qilu Hospital of Shandong University, Jinan Shandong, China, Tel: (0351)82169636 Fax: (0531)86109975; E-mail: grc7636@126.com

Received October 23, 2012; Accepted October 27, 2012; Published October 31, 2012

Citation: Guiyan Y, Rui Z, Benjie W, Chunmin W, Xiaoyan L, et al. (2012) Determination of Rocuronium in Human Plasma by High Performance Liquid Chromatography-Tandem Mass Spectrometry and its Pharmacokinetics in Patients. J Bioequiv Availab 4: xxix-xxx. doi:10.4172/jbb.10000e23

Copyright: @ 2012 Guiyan Y, et al. This is an open-access article distributed under the terms of the Creative Commons Attribution License, which permits unrestricted use, distribution, and reproduction in any medium, provided the original author and source are credited. 
Citation: Guiyan Y, Rui Z, Benjie W, Chunmin W, Xiaoyan L, et al. (2012) Determination of Rocuronium in Human Plasma by High Performance Liquid Chromatography-Tandem Mass Spectrometry and its Pharmacokinetics in Patients. J Bioequiv Availab 4: xxix-xxx. doi:10.4172/ jbb.10000e23

\section{Sample preparation}

A $25 \mu \mathrm{L}$ aliquot of the I.S. solution $(10 \mu \mathrm{g} / \mathrm{mL})$ and $300 \mu \mathrm{L}$ mixture of methanol/acetonitrile $(1 / 1, \mathrm{v} / \mathrm{v})$ were added to $200 \mu \mathrm{L}$ of plasma samples, mixed on a Multi-Tube Vortexer VX-02 (TARGIN TECH CO., Ltd) for 1 minute, followed by 5 minutes centrifugation at $11132 x$ g. A $5 \mu \mathrm{L}$ of upper organic layer was injected for HPLC/MS/MS analysis.

\section{Pharmacokinetic study design}

Seven patients, who will undergo a surgery using rocuronium as anesthetic, signed the informed consent form after understanding the content, rights, obligations and risks. $4 \mathrm{~mL}$ blood samples were collected in heparinized tubes before (0) and 2, 4, 9, 15, 25, 40, 60, $90,120,180,240$, and $360 \mathrm{~min}$ after hocused. Blood samples were centrifuged at $1752 \mathrm{x}$ g for 10 minutes and plasma was subsequently quoted and stored at $-20^{\circ} \mathrm{C}$ until analyzed. Drug and Statistic (DAS, version 2.0, by Chen et al. [6], China) was used to calculate the main pharmacokinetic parameters as half life $\left(\mathrm{t}_{1 / 2}\right)$ and area under the plasma concentration versus time curve $\left(\mathrm{AUC}_{0-24}\right.$ and $\left.\mathrm{AUC}_{0-\infty}\right)$. The peak plasma concentration $\left(\mathrm{C}_{\max }\right)$ and its corresponding time $\left(\mathrm{T}_{\max }\right)$ were observed values.

\section{Results}

\section{Method validation}

Specificity was assessed by analyzing six different human blank plasma samples.

Typical chromatograms are shown in Figure 1. Matrix effects were evaluated by signal response comparison of six extracted blank plasma samples with those of six analytical standards (at three concentration levels of QCs). No matrix effects were detected in the study.

The calibration curves were constructed by analyzing five independent standard plasma samples. The peak area ratios of rocuronium and IS were measured and plotted against the concentrations of rocuronium spiked with blank plasma, and the least square method with $1 / \mathrm{x}^{2}$ weighting factor was used. The weighted regression equation was $\mathrm{y}=12.2106 \mathrm{x}+0.0354$ with a correlation coefficient $r^{2}$ of 0.9976 . It was found to be linear over the range of $5-3000 \mu \mathrm{g} / \mathrm{mL}$.

The extraction recoveries of rocuronium were determined by comparing the mean peak areas of the extracted QC samples at 3 concentrations of $10,100,2000 \mu \mathrm{g} / \mathrm{mL}$ with mean peak areas of appropriate concentrations of standard solutions. The results were found to be $92.0,92.9$ and $92.6 \%$ with a precision (RSD) of $1.68,0.71$ and $1.22 \%$, respectively.

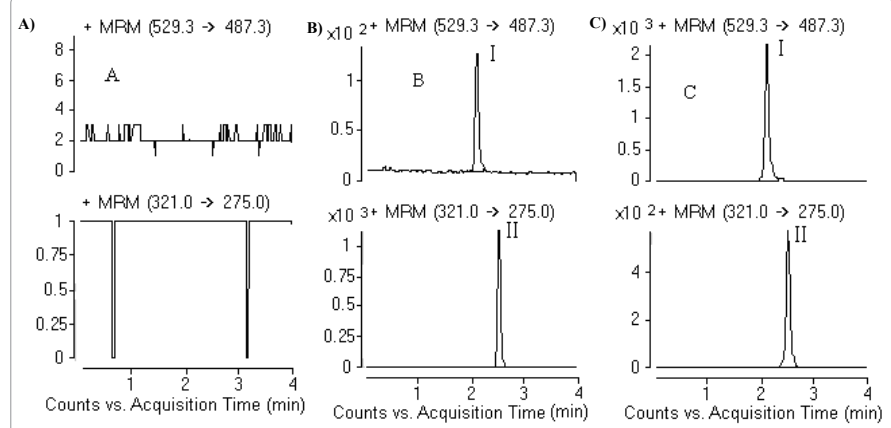

Figure 1: a) Chromatograms of blank plasma. B) Plasma spiked with rocuronium and IS standard, c) Extracted plasma sample from a patient spiked with IS ( $\alpha$-rocuronium, $\alpha-I S$ ).
Precision and accuracy were assessed investigating. QC samples on three different validation days. The intra-day precision (RSD) ranged between 1.10 and $1.95 \%$, while the inter-day precision (RSD) ranged between 3.97 and $8.05 \%$.

The freeze-thaw and freeze stabilities of rocuronium were evaluated by analyzing QC samples undergoing two freeze-thaw cycles $\left(-20^{\circ} \mathrm{C}\right.$ to room temperature) and placing QC samples at $-20^{\circ} \mathrm{C}$ for $24 \mathrm{~h}$ and 7 days. The stability results suggested that rocuronium was stable under these storage and processing conditions.

\section{Pharmacokinetic study}

The concentration of rocuronium in patient's plasma was determined using the established LC-MS/MS method. The main pharmacokinetic parameters of rocuronium were as follows: $\mathrm{t}_{1 / 2}=78.18 \pm 26.88 \mathrm{~min}, \mathrm{~T}_{\max }=2.29 \pm 0.76 \mathrm{~min}, \mathrm{C}_{\max }=27.99 \pm 48.93 \mu \mathrm{g} /$ $\mathrm{mL}, \mathrm{AUC}_{0-360}=276.93 \pm 220.56 \mu \mathrm{g} / \mathrm{mL} \cdot \mathrm{h}, \mathrm{AUC}_{0-\infty}=279.67 \pm 220.29$ $\mu \mathrm{g} / \mathrm{mL} \cdot \mathrm{h}$.

\section{Conclusion}

A sensitive and selective LC-MS method was developed and validated for the determination of rocuronium in human plasma. This has been successfully applied to pharmacokinetic studies in patients who were hocused using rocuronium.

\section{References}

1. Wierda JM, de Wit AP, Kuizenga K, Agoston S (1990) Clinical observations on the neuromuscular blocking action of Org 9426, a new steroidal nondepolarizing agent. Br J Anaesth 64: 521-523.

2. Levy JH, Davis GK, Duggan J, Szlam F (1994) Determination of the hemodynamics and histamine release of rocuronium (Org 9426) when administered in increased doses under $\mathrm{N}_{2} \mathrm{O} / \mathrm{O}_{2}$-sufentanil anesthesia. Anesth Analg 78: 318-321.

3. Yu Ge, Gao Ling, Yang Xiaofeng (2006) Gas chromatographic mass spectrometric assay for rocuronium in human plasma. International journal of anesthesiology and resuscitation 27: 72-74

4. Shi Weidong, Li Haibo, Zhang Ruiqin, Li Dehui, Wang Hongliang (2005) Determination of rocuronium in patient plasma who will undergo heart valve replacement/suture surgery under extracorporeal circulation. Chinese Journal of Anesthesiology 25: 237-238.

5. Farenc C, Enjalbal C, Sanchez P, Bressolle F, Audran M, et al. (2001) Quantitative determination of rocuronium in human plasma by liquid chromatography-electrospray ionization mass spectrometry. J Chromatogr A 910: 61-67.

6. Chen ZY, Zheng QS, Sun RY (2002) Functions of the DAS software for pharmacological calculation. Chinese Journal of Clinical Pharmacology and Therapeutics 7: 502-504 